\title{
Interacciones farmacológicas potenciales en pacientes ambulatorios en expedientes de queja médica de la CONAMED
}

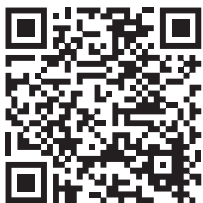

\author{
Potential drug interactions in outpatients in \\ CONAMED medical complaint records
}

José Luis Garduño Hernández*

\section{RESUMEN}

Introducción: Las interacciones farmacológicas son un tipo de error de medicación que pueden provocar un daño al paciente. Éstas deben ser detectadas durante la validación de la prescripción y corregidas por el profesional encargado en la atención del paciente. El propósito de este estudio fue la identificación, clasificación y la prevalencia de interacciones farmacológicas, así como los medicamentos involucrados en consultas ambulatorias de pacientes con expediente de queja médica de la Comisión Nacional de Arbitraje Médico (CONAMED). Material y métodos: Se realizó un estudio observacional descriptivo, retrospectivo y transversal. Se revisaron las prescripciones de consultas ambulatorias de los expedientes de queja médica del año 2013 al 2016 registrados en el sistema de farmacoterapia de la CONAMED. Resultados: Se analizaron las prescripciones de 1,995 consultas en 170 expedientes clínicos de queja médica. Sólo 1,720 cumplieron con los criterios de inclusión. El número promedio de medicamentos prescritos por consulta para el sexo femenino fue de $5.1 \pm 3.5(p<0.01)$, y para pacientes $\geq 65$ años fue de $6.4 \pm 3.8$ ( $p<0.01)$. El número de consultas con polifarmacia (cinco o más medicamentos) en pacientes $\geq$ a 65 años fue de 305 (63.9\%). El número de interacciones detectadas según la gravedad fueron moderadas 1,415 (48.5\%), mayores 1,253 (43\%), menores 193 (6.6\%) y contraindicadas 54 (1.9\%), y de acuerdo con su mecanismo de acción el 46.8\% corresponden a interacciones farmacodinámicas y el 32.1\% farmacocinéticas. Los medicamentos mayormente involucrados en las interacciones son el bezafibrato, pravastatina, captopril, metformina, ácido acetilsalicílico, diclofenaco, insulina y enalapril. Conclusión: Se analizaron las interacciones farmacológicas de un total de 8,282 medicamentos prescritos en consultas ambulatorias de expedientes de queja médica. Las interacciones farmacológicas se presentan con mayor frecuencia en pacientes mayores de 65 años con polimedicación y en el género femenino. Las mayoritarias son aquéllas que afectan a la farmacodinamia. Se debe realizar la búsqueda de interacciones farmacológicas con el uso de herramientas informáticas durante la prescripción para una mejora en el tratamiento y seguridad del paciente.

Palabras clave: Interacciones farmacológicas, polifarmacia, farmacoterapia, errores de medicación, evento adverso, seguridad del paciente.

\footnotetext{
ABSTRACT

Introduction: Drug interactions are a type of medication error that can cause harm to the patient. These must be detected during the validation of the prescription and corrected by the professional in charge of the patient care. The purpose of this study was the identification, classification and prevalence of drugs interactions, as well as the medications involved in outpatient consultations of patients with a medical complaint file from the National Medical Arbitration Commission (CONAMED). Material and methods: A descriptive, retrospective and cross-sectional observational study was carried out. The outpatient consultation prescriptions of the medical complaint files registered from 2013 to 2016 in the CONAMED pharmacotherapy system were reviewed. Results: 1,995 consultations were analyzed in 170 medical records. Only 1,720 met the inclusion criteria. The number of medications per consultation for the female
}

* Pasante de la carrera de Química Farmacéutica Biológica en la UNAM y estudiante de segundo año de la carrera de Médico Cirujano y Partero en el IPN.

Correspondencia: JLGH, qfbpi1184@gmail.com Conflicto de intereses: Declaro que no existe conflicto de intereses. Citar como: Garduño $\mathrm{HJL}$. Interacciones farmacológicas potenciales en pacientes ambulatorios en expedientes de queja médica de la CONAMED. Rev CONAMED 2020; 25(3): 115-122. https://dx.doi. org/10.35366/95983

Financiamiento: Ninguno.

Recibido: 12/05/2020. Aceptado: 04/08/2020. 
sex was $5.1 \pm 3.5$ ( $p<0.01)$. The average number of medications per consultation for patients $\geq 65$ years was $6.4 \pm 3.8$ ( $p<0.01)$. The number of consultations with 5 or more medications (polypharmacy) in patients older than 65 or equal to 65 years was 305 (63.9\%). The number of detected interactions according to severity were moderate 1415 (48.5\%), higher 1,253 (43\%), minor 193 (6.6\%) and contraindicated 54 (1.9\%), and according to their mechanism of action 46.8\% correspond to pharmacodynamic interactions and $32.1 \%$ pharmacokinetics. The drugs most involved in the interactions are bezafibrate, pravastatin, captopril, metformin, acetylsalicylic acid, diclofenac, insulin and enalapril. Conclusion: Pharmacological interactions of a total of 8,282 medications prescribed in outpatient medical complaint records were analyzed. Pharmacological interactions occur more frequently in patients older than 65 years with polymedication and in the female gender. The majority are those that affect pharmacodynamics. The search for drug interactions with the use of softwares during the prescription should be carried out to improve treatment and patient safety.

Keywords: Drug interactions, polypharmacy, pharmacotherapy, medication errors, adverse event, patient safety.

\section{INTRODUCCIÓN}

En México el crecimiento de la morbilidad de enfermedades crónicas aumenta el uso conjunto de dos o más medicamentos como parte del manejo integral de estos padecimientos. En el 2018, las enfermedades crónicas no transmisibles como las enfermedades del corazón, la diabetes mellitus, los tumores malignos, las enfermedades cerebrovasculares y enfermedades pulmonares obstructivas crónicas ocuparon los primeros cinco lugares de mortalidad en México en personas de 65 o más años.' La concomitancia de pluripatologías que es común en este grupo etario obliga al uso de un conjunto de medicamentos para tratar dichas enfermedades, aumentando la probabilidad de que se presente una interacción farmacológica. ${ }^{2-4}$

Las interacciones farmacológicas surgen cuando los efectos de un medicamento se alteran por la administración conjunta de otro medicamento.

Muchos factores determinan la respuesta clínica observada, incluidas las propiedades farmacológicas del tratamiento y las condiciones como edad, género y comorbilidades; además, los procesos de liberación, absorción, distribución, metabolismo y eliminación (LADME) de un medicamento influyen en la biodisponibilidad del fármaco. La eficacia terapéutica puede verse influida tanto negativamente como positivamente en la alteración de alguno de estos procesos. De manera similar, el efecto de un fármaco puede verse alterado en su farmacodinamia si se prescribe en conjunto con otro que actúe sobre el mismo receptor o sistema fisiológico, antagonizando el efecto del primero o realizando un sinergismo exacerbado llevando a una intoxicación. ${ }^{5}$

Las interacciones pueden clasificarse en dos formas: según el mecanismo de acción de la interacción y según la gravedad. ${ }^{6}$
Según el mecanismo de acción, las interacciones farmacológicas se clasifican en:

- Farmacocinéticas; ocurren cuando los procesos de liberación, absorción, distribución, metabolismo y eliminación (LADME) del principio activo (fármaco) de un medicamento se ven afectados por otro fármaco o medicamento. La principal causa de estas interacciones es la alteración de la eliminación del principio activo (debido a la interferencia con el metabolismo hepático, la excreción renal o el transporte transcelular).

- Farmacodinámicas; se producen cuando los fármacos prescritos conjuntamente actúan sobre los mismos receptores o sistemas fisiológicos, obteniendo un efecto antagónico o sinérgico. ${ }^{5}$

De acuerdo con la gravedad del efecto adverso generado por la interacción farmacológica, la International Business Machines (IBM) Micromedex Drug Interactions lo clasifica de la siguiente manera:

- Contraindicada: los fármacos están contraindicados para su uso simultáneo.

- Mayor o importante: la interacción puede causar la muerte y/o requerir intervención médica para minimizar o evitar efectos adversos graves.

- Moderada: la interacción puede agravar la condición del paciente y/o requerir una alteración de la terapia.

- Menor o secundaria: la interacción puede tener efectos clínicos limitados. Los síntomas pueden incluir un aumento de la frecuencia o la gravedad de los efectos adversos pero, en general, no requerían una alteración importante de la terapia.? 
Hay diferentes estudios que demuestran que hay una alta incidencia de la presencia de interacciones farmacológicas en diferentes niveles de atención, aunado a las enfermedades crónicas en pacientes adultos mayores, los cuales tienen que hacer uso de la polifarmacia, definida como el uso en conjunto de cinco o más medicamentos, ${ }^{4,8}$ esto debido a pluripatologías que llega a padecer este grupo u otros, aumentando el riesgo de una interacción farmacológica.

La frecuencia de aparición de interacciones farmacológicas y efectos adversos en pacientes con polifarmacia es mayor cuanto más elevado sea el número de medicamentos que se administran de forma conjunta. Se estima que los pacientes que utilizan dos medicamentos en su tratamiento tienen alrededor de un 6\% de probabilidad de presentar efectos adversos debido a una interacción farmacológica en su tratamiento, aumentado alrededor de 50\% cuando usan seis y casi un 100\% cuando son ocho o más. ${ }^{9,10}$ Un estudio en Perú en pacientes ambulatorios encontró una correlación positiva y moderada (Rho = 0.315) entre el número de medicamentos prescritos y la cantidad de interacciones farmacológicas, por lo que, en la medida en que aumenta el número de medicamentos prescritos, es probable que aumente la probabilidad de padecer un efecto adverso por alguna interacción farmacológica."

Otro ejemplo es el trabajo realizado en un hospital del ISSSTE en Pachuca, Hidalgo, México, con prescripciones de pacientes del servicio de consulta externa, en quienes se identificó que las prescripciones con cuatro medicamentos diferentes cubrían un $28.13 \%$ del total de interacciones encontradas, ${ }^{12}$ mientras que otro estudio realizado en Venezuela encontraron en prescripciones de pacientes hospitalizados que las prescripciones con uno a cinco medicamentos abarcaba el $44.20 \%$ del total de interacciones encontradas. ${ }^{13}$

En el presente trabajo, se realizó la búsqueda de interacciones farmacológicas en las prescripciones de consultas ambulatorias de expedientes de queja médica del año 2013 al 2016 registrados en el sistema de farmacoterapia de la CONAMED. Las interacciones se identificaron con el software IBM Micromedex Drug Interactions ${ }^{\circledR}$. Éstas se clasificaron de acuerdo con su gravedad y mecanismo de interacción, determinando así su prevalencia.

\section{MATERIAL Y MÉTODOS}

Se trata de un estudio retrospectivo, transversal, descriptivo. Se revisaron las prescripciones médicas de consultas ambulatorias de los expedientes clínicos de queja médica del año 2013 al 2016 registrados en la base de datos del sistema de farmacoterapia de la CONAMED. ${ }^{14}$ Los criterios de inclusión fueron: expedientes clínicos de queja médica de pacientes mayores de 18 años, con prescripciones en consultas ambulatorias con dos o más medicamentos alopáticos. Para el análisis se consideró edad, género, medicamento prescrito, número de medicamentos por consulta, mecanismo de acción de la interacción y su gravedad. El conjunto de medicamentos prescritos por consulta se ingresó en el software IBM Micromedex Drug Interactions ${ }^{\circledR}$ para determinar la existencia de interacciones farmacológicas, se revisó el mecanismo de ésta, farmacocinético o farmacodinámico y su clasificación según la gravedad de la interacción en menor, moderada, mayor o contraindicada. Con los datos se elaboró una base de datos en Microsoft Excel ${ }^{\circledR} 2018$.

Se analizó el número de medicamentos e interacciones en la prescripción de cada consulta. Se estratificó por grupo de edad; menores de 65 años y 65 años o más, así como por género. Se realizaron pruebas de contraste de hipótesis entre las medias para determinar si existía diferencia significativa entre los grupos. Se realizó una correlación de Spearman para determinar si había relación entre el número de fármacos prescritos y el número de interacciones por consulta.

\section{RESULTADOS}

Se analizaron las prescripciones de 1,995 consultas en 170 expedientes clínicos de queja médica, de las cuales 1,720 cumplieron con los criterios de inclusión, 86\% del universo estudiado. El promedio de edad de los pacientes fue de $54.7 \pm 16.3$ años.

Se determinó el promedio de medicamentos prescritos por consulta del total, y estratificado por grupo etario y género. Se realizó lo mismo para el promedio de interacciones por consulta (Tabla 7). 
Se detectaron un total de 2,915 interacciones farmacológicas en las 1,720 consultas analizadas y se clasificaron de acuerdo con su gravedad, de las cuales 54 (1.9\%) fueron contraindicadas, 1,253 (42\%) mayores, 1,415 (48.5\%) moderadas y 193 (6.6\%) menores (Figura 7).

Se encontró que 33 consultas de las 1,720 analizadas contenían interacciones de tipo contraindicadas en su prescripción, 557 consultas contenían interacciones mayores, 583 moderadas y 142 menores. Sólo 405 consultas no presentaron algún tipo de interacción en su prescripción (Figura 2).

Se contabilizaron las consultas de acuerdo con el grupo etario y subdividiendo éste en grupos dependiendo del número de interacciones encontradas en la prescripción (Tabla 2).

Se determinó la prevalencia del número de consultas con polifarmacia por grupo de edad (Tabla 3).

Se realizó una correlación de Spearman con el número de medicamentos prescritos por consulta y la aparición de interacciones farmacológicas para determinar si existe una relación entre éstos. Se obtuvo una Rho $=0.683$, con una correlación positiva moderada fuerte entre el número de medicamentos prescritos y el número de interacciones encontradas por consulta.

\begin{tabular}{lcc}
\multicolumn{2}{c}{$\begin{array}{c}\text { Tabla 1: Número de medicamentos e } \\
\text { interacciones involucrados por consulta } \\
\text { para cada género y grupo de edad. }\end{array}$} \\
\hline \multicolumn{2}{c}{ Promedio } \\
\hline Variable & $4.8 \pm 3.0$ & \\
\hline Medicamentos prescritos & $4.5 \pm 2.5$ & \\
por consulta & $5.1 \pm 3.5$ & $\mathrm{p}<0.01$ \\
Sexo masculino & $4.2 \pm 2.4$ & \\
Sexo femenino & $6.4 \pm 3.8$ & $\mathrm{p}<0.01$ \\
Menores de 65 años & $1.7 \pm 3.1$ & \\
65 años o más & & \\
Interacciones encontradas & $1.3 \pm 2.1$ & \\
por consulta & $2.1 \pm 3.8$ & $\mathrm{p}<0.01$ \\
Sexo masculino & $1.4 \pm 2.7$ & \\
Sexo femenino & $2.4 \pm 3.9$ & $\mathrm{p}<0.01$ \\
\hline Menores de 65 años & & \\
65 años o más & & \\
\hline
\end{tabular}

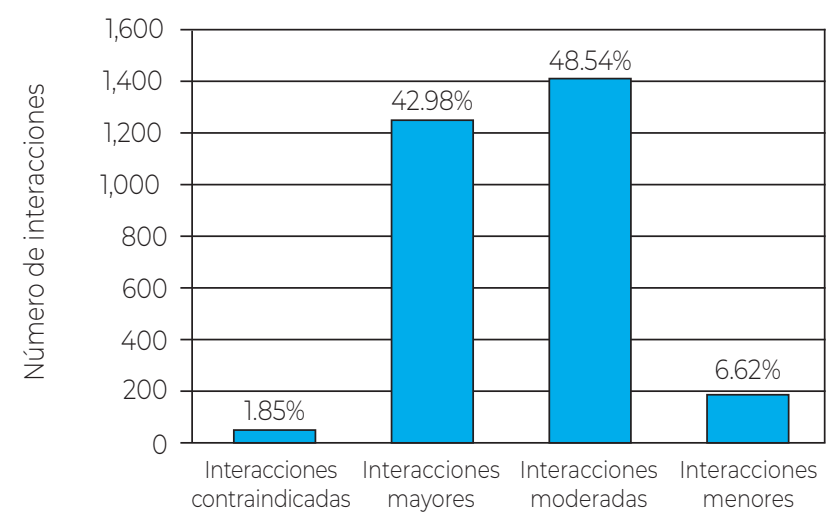

Figura 1: Prevalencia por gravedad de las interacciones encontradas. Número de interacciones detectadas y clasificadas según su gravedad por medio del software IBM Micromedex Drug Interactions ${ }^{\circledR}$ en 1,720 consultas de expedientes de queja médica de la CONAMED.

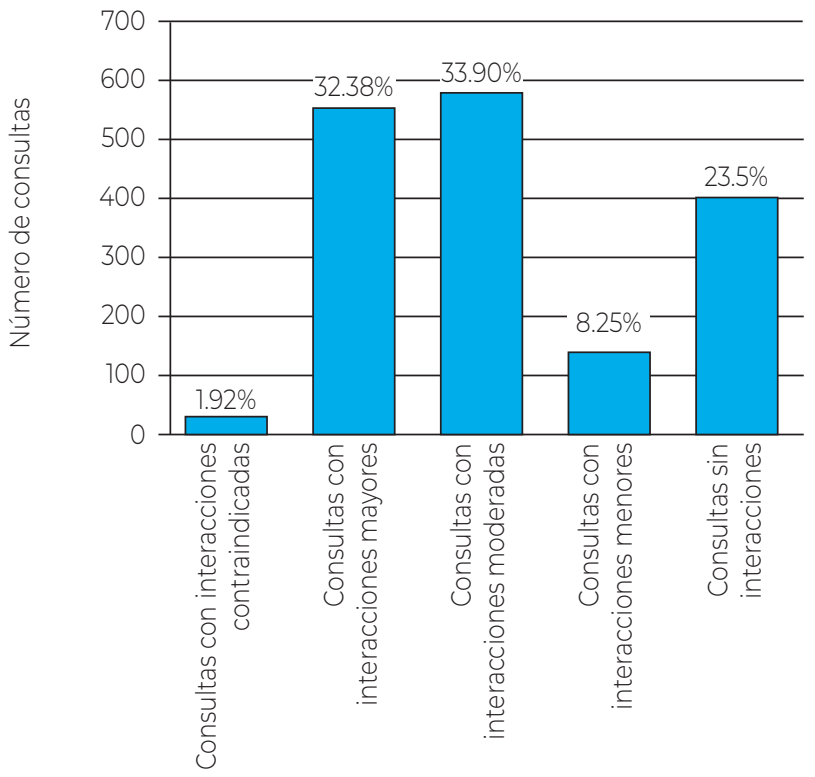

Figura 2: Prevalencia de la interacción por consulta prestada. Clasificación de las 1,720 consultas de acuerdo con el tipo de interacción farmacológica encontrada en su prescripción.

También se encontró que el 32.1\% de las interacciones son de tipo farmacocinéticas, el $46.8 \%$ son interacciones farmacodinámicas y el $21.1 \%$ corresponden a interacciones cuyo mecanismo de acción es desconocido.

En la Tabla 4 se presentan las primeras 20 interacciones farmacológicas con mayor prevalencia halladas en este trabajo, con su respectiva gravedad y mecanismo de acción. 


\begin{tabular}{lcc}
$\begin{array}{l}\text { Tabla 2: Prevalencia del número de interacciones } \\
\text { involucradas por consulta para cada grupo de edad. }\end{array}$ \\
\hline Variable & $n$ & $\%$ \\
\hline Consultas de pacientes & 1,243 & 100.0 \\
menores de 65 años & & \\
Sin interacciones & 670 & 53.9 \\
Con 1 interacción & 239 & 19.2 \\
Con 2 interacciones & 106 & 8.5 \\
Con 3 interacciones & 64 & 5.1 \\
Con 4 interacciones & 47 & 3.8 \\
Con 5 más interacciones & 117 & 9.4 \\
Consultas de pacientes & 477 & 100.0 \\
de 65 años o más & & \\
Sin interacciones & 202 & 42.3 \\
Con 1 interacción & 84 & 17.6 \\
Con 2 interacciones & 48 & 10.1 \\
Con 3 interacciones & 27 & 5.7 \\
Con 4 interacciones & 37 & 7.8 \\
Con 5 o más interacciones & 79 & 16.6 \\
\hline
\end{tabular}

\section{DISCUSIÓN}

El promedio de medicamentos por consulta (Tabla 1) fue mayor para el sexo femenino $(5.1 \pm 3.5)$ que para el masculino ( $4.5 \pm 2.5)$, evidenciado que las mujeres están más expuestas a presentar un mayor número de enfermedades. Tan sólo en el 2019 en México las mujeres presentaron mayor morbilidad en enfermedades infecciones respiratorias agudas, infección en vías urinarias, infecciones intestinales además de enfermedades crónicas como obesidad, hipertensión arterial y diabetes mellitus no insulinodependiente (tipo II).' En el caso del grupo etario, el número promedio de medicamentos por consulta fue mayor para pacientes igual o mayores de 65 años (6.4 \pm 3.8) que para pacientes menores de 65 (4.2 \pm 2.4$)$. El número de interacciones encontradas por consulta, también presentado en la Tabla 7, fue mayor para las mujeres $(2.1 \pm 3.89)$ que para los hombres $(1.3 \pm 2.1)$ y mayor para el grupo de 65 años o más $(2.4 \pm 3.9)$ que para los menores de 65 años (1.4 \pm 2.7$)$. Relacionando estos resultados con los anteriores, se puede observar que a mayor cantidad de medicamentos prescritos por consulta, mayor probabilidad de encontrar interacciones farmacológicas. La vulnerabilidad a las interacciones farmacológicas en el anciano se potencializa por la coexistencia de enfermedades y la necesidad de la utilización simultánea de varios medicamentos. ${ }^{15}$ Además, se ha reportado una prevalencia de interacciones farmacológicas en pacientes ancianos del 35 al 60\% de las cuales un 5 a 15\% de éstos se presentan con significancia clínica. ${ }^{2}$ En el trabajo de Ribes Moya C., se encontró que tras el análisis estadístico por género de las interacciones potenciales detectadas en prescripciones dispensadas en una farmacia durante un año, el 63.64\% se produjeron en mujeres, y en el análisis por grupo etario, más del 63\% se producen en pacientes mayores de 56 años, ${ }^{16}$ lo que reafirma que a mayor edad hay mayor probabilidad de encontrar alguna interacción farmacológica en la prescripción, mientras que para el género femenino ocurre lo mismo.

En el número de interacciones encontradas por consulta estratificados por grupo etario mostrado en la Tabla 2, se puede hacer notar que en el grupo menores de 65 años es mayor el número de consultas totales ( $n=1,245$ ) que el de 65 años o más ( $n=477$ ). En los dos grupos la mayor prevalencia se encuentra en las consultas sin interacciones con 53.9\% para el primero y $42.3 \%$ para el segundo grupo; sin embargo, a pesar de que el tamaño de muestra difiere, se observa una mayor tendencia a la alta en el porcentaje de interacciones por consulta para el grupo de 65 años o más, tan sólo las consultas con cinco o más interacciones para el primer grupo es de $9.4 \%$ y para el segundo es de

\begin{tabular}{|c|c|c|}
\hline \multicolumn{3}{|c|}{$\begin{array}{l}\text { Tabla 3: Prevalencia de polifarmacia } \\
\text { por grupo de edad. }\end{array}$} \\
\hline Variable & $\mathrm{n}$ & $\%$ \\
\hline $\begin{array}{l}\text { Consultas de pacientes } \\
\text { menores de } 65 \text { años }\end{array}$ & 1,243 & 100.0 \\
\hline Con polifarmacia & 415 & 33.4 \\
\hline Sin polifarmacia & 828 & 66.6 \\
\hline $\begin{array}{l}\text { Consultas de pacientes de } \\
65 \text { años o más }\end{array}$ & 477 & 100.0 \\
\hline Con polifarmacia & 305 & 63.9 \\
\hline Sin polifarmacia & 172 & 36.1 \\
\hline
\end{tabular}


Tabla 4: Interacciones con mayor prevalencia encontradas con su respectiva gravedad.

\begin{tabular}{lccll}
\hline \multicolumn{1}{c}{ Combinación de fármacos } & Frecuencia & Gravedad & Mecanismo \\
\hline Bezafibrato & Pravastatina & 80 & Mayor & Interferencia en metabolismo \\
Captopril & Metformina & 78 & Moderada & Desconocido \\
Ácido acetilsalicílico & Metformina & 72 & Mayor & Sinergismo hipoglucemiante \\
Ácido acetilsalicílico & Diclofenaco & 65 & Mayor & Antagonismo en efecto antiplaquetario \\
Insulina & Metformina & 59 & Moderada & Sinergismo hipoglucemiante \\
Enalapril & Metformina & 58 & Moderada & Desconocido \\
Ácido acetilsalicílico & Enalapril & 56 & Moderada & Sinergismo vasodilatador \\
Ácido acetilsalicílico & Insulina & 52 & Moderada & Desconocido \\
Enalapril & Glibenclamida & 49 & Moderada & Desconocido \\
Enalapril & Insulina & 44 & Moderada & Desconocido \\
Ácido acetilsalicílico & Furosemida & 41 & Mayor & Antagonismo diurético \\
Captopril & Glibenclamida & 41 & Moderada & Desconocido \\
Ácido acetilsalicílico & Clibenclamida & 40 & Mayor & Sinergismo hipoglucemiante \\
Ácido acetilsalicílico & Pentoxifilina & 39 & Mayor & Desconocido \\
Captopril & Diclofenaco & 39 & Moderada & Disminución de biodisponibilidad \\
Diclofenaco & Fluoxetina & 39 & Mayor & Antagonismo antiplaquetario \\
Diclofenaco & Lidocaína/ & 39 & Mayor & Sinergismo, mayor probabilidad de \\
Enalapril & hidrocortisona & & & sangrado gástrico \\
Ácido acetilsalicílico & Furosemida & 39 & Moderada & Sinergismo antihipertensivo \\
Bezafibrato & Metoprolol & 36 & Moderada & Antagonismo antihipertensivo \\
\hline & Insulina & 36 & Moderada & Desconocido \\
\hline
\end{tabular}

16.6\%, lo que reafirma que los pacientes mayores de 65 años están más expuestos a padecer interacciones farmacológicas.

En la prevalencia de la polifarmacia estratificada por grupo etario mostrada en la Tabla 3, se observa que en los pacientes menores de 65 años el $33.4 \%$ de las consultas contiene polifarmacia en su prescripción, mientras que para los pacientes de 65 años o más es del 63.9\%, esto último continúa reafirmando el hecho de que en este grupo de pacientes hay una mayor probabilidad de encontrar interacciones farmacológicas en su prescripción. Este mayor riesgo también viene determinado por factores dependientes del paciente, de la polifarmacia y de las dificultades de comunicación entre los diferentes profesionales de la salud que atienden el mismo caso. ${ }^{4}$ En un estudio realizado en el 2010 por López-Picazo y colaboradores en expedientes clínicos digitalizados de consultas ambulatorias en España, con una $n=430,525$ sujetos de estudio, se encontró una mayor probabilidad de aparición de una interacción farmacológica entre personas con afecciones crónicas, ancianos, mujeres y sujetos polimedicados. ${ }^{3}$ Esto último coincide con los resultados antes mencionados.

Las interacciones moderadas fueron las de mayor prevalencia con un $48.54 \%$ como se muestra en la Figura 7, seguido por poco de las mayores con un $42.98 \%$ y con mayor diferencia, las menores con $6.62 \%$ y contraindicadas con $1.85 \%$, estas últimas son las más importantes debido a su potencial daño. En el trabajo de Gómez Olivan y Hernández C., las interacciones farmacológicas de gravedad moderada fueron las de mayor prevalencia, seguida de las mayores, similar al resultado obtenido.12

De 1,720 consultas analizadas, en el $76.45 \%$ se encontró alguna interacción farmacológica en su prescripción. Lasconsultasqueensus prescripciones 
se hallaron interacciones moderadas son las de mayor prevalencia con un 33.90\% (Figura 2), seguidas de las que contienen interacciones mayores con 32.38\%, consultas con interacciones menores con un $8.25 \%$, y en último lugar están las que contienen interacciones contraindicadas con $1.92 \%$. El 23.5\% de las consultas no presenta ninguna interacción farmacológica en su prescripción, a diferencia del trabajo de Gómez Olivan y Hernández C., en donde el tamaño de muestra es de 80,731 prescripciones, ellos encontraron que tan sólo el 2.53\% presentan interacciones farmacológicas potenciales. ${ }^{12}$

Se encontró una correlación positiva moderada fuerte de Spearman (Rho = 0.68), esto se puede traducir en que a mayor cantidad de medicamentos prescritos, mayor será la probabilidad de que se presente alguna interacción farmacológica. En el trabajo realizado por Abner A. y Rosa Chabely C., se encontró una correlación positiva moderada (Rho $=0.315)$ entre el número de medicamentos prescritos y el número de interacciones farmacológicas encontradas en prescripciones de recetas de pacientes ambulatorios," coincidiendo con el resultado obtenido en el análisis de consultas ambulatorias en expedientes de queja médica de la CONAMED.

Las interacciones farmacodinámicas resultaron ser las más prevalentes con un 46.8\% del total, seguidas de las farmacocinéticas con un $32.1 \%$. El $21.1 \%$ de interacciones fueron desconocidas. En el trabajo de Ramez Constantino C., encontraron que las interacciones farmacodinámicas correspondían a un 66\% y las farmacocinéticas a un 35\% del total de interacciones encontradas. Ribes Moya C., reportó que las interacciones farmacodinámicas a parecieron en una mayor proporción frente a las farmacocinéticas, 57.94 y $41.12 \%$ respectivamente. ${ }^{13,16}$ Estos resultados ponen de manifiesto que las interacciones farmacodinámicas son las más frecuentes.

En la Tabla 4 se muestran las combinaciones de medicamentos que pueden presentar interacción halladas con mayor frecuencia en este trabajo. La combinación de bezafibrato y pravastatina fue la de mayor prevalencia, el uso concomitante de estos fármacos incrementa la incidencia y la severidad de miopatía debido a un incremento en la concentración plasmática de pravastatina, esto debido a una interacción en su metabolismo. ${ }^{17}$ Se puede hacer notar que las interacciones más frecuentes involucran medicamentos como el ácido acetilsalicílico, enalapril, insulina, captopril, glibenclamida, metformina, y diclofenaco. Estos fármacos, utilizados para tratar enfermedades crónico-degenerativas, como la diabetes mellitus (tipo II) y la hipertensión arterial sistémica, se tienen que utilizar en combinación con más medicamentos aumentando la probabilidad de manifestar algún evento adverso debido a la interacción farmacológica y por lo tanto una falla en el tratamiento. Se ha documentado el efecto hipoglucemiante por el ácido acetilsalicílico debido la disminución de la producción de glucosa hepática, 18,19 aunado a esto, la combinación de ácido acetilsalicílico con algún hipoglucemiante tiene un efecto sinérgico, pudiéndose manifestar hipoglucemia como un evento adverso. En el trabajo de Gómez Oliván y colaboradores, la mayor prevalencia de interacciones farmacológicas encontrada fue entre antiinflamatorios no esteroideos e inhibidores de la enzima convertidora de angiotensina (20.8\%), seguido de antiinflamatorios no esteroideos con betabloqueadores (12.7\%) y entre los inhibidores de la enzima convertidora de angiotensina y diuréticos de asa (12.4\%)..$^{2}$ A diferencia de este trabajo, no se encuentra dentro de su lista de interacciones farmacológicas la pravastatina ni el bezafibrato; sin embargo, reportan que los medicamentos mayormente involucrados en interacciones farmacológicas son aquéllos que sirven para tratar enfermedades crónico-degenerativas, coincidiendo con lo antes ya mencionado.

\section{CONCLUSIÓN}

Las interacciones farmacológicas potenciales pueden manifestarse como un efecto adverso en el paciente, traduciéndose en una serie de complicaciones clínicas llegando a poner en riesgo la vida. El género femenino y el grupo etario de 65 años o más son los más propensos a presentar una interacción farmacológica en su prescripción. El aumento de la cantidad de fármacos utilizados en tratamiento está directamente relacionado con la aparición de una interacción farmacológica, por lo que hay que hacer énfasis en pacientes con polifarmacia. La búsqueda de interacciones farmacológicas durante la prescripción se puede 
realizar utilizando herramientas informáticas, mejorando la calidad del tratamiento y la seguridad del paciente.

\section{BibliografíA}

1. Dirección General de Epidemiología. Anuario de morbilidad 1984-2019 [Base de datos en Internet]. [Consultado 11 de julio de 2020] Disponible en: http://www. epidemiologia.salud.gob.mx/anuario/html/anuarios.html

2. Magro L, Moretti U, Leone R. Epidemiologyand characteristics of adverse drug reactions caused by drugdrug interactions. Expert Opin Drug Saf. 2012; 11 (1): 83-94.

3. López-Picazo JJ, Ruiz JC, Sánchez JF, Ariza A, Aguilera B, Lazaro D et al. Prevalence and typology of potential drug interactions occurring in primary care patients. Eur J Gen Pract. 2010; 16 (2): 92-99.

4. Iniesta-Navalón C, Urbieta-Sanz E, Gascón-Cánovas JJ. Análisis de las interacciones medicamentosas asociadas a la farmacoterapia domiciliaria en pacientes ancianos hospitalizados. Rev Clin Esp. 2011; 211 (7): 344-351.

5. Brewer L, Williams D. Clinically relevant drug-drug and drug-food interactions: underlying mechanisms and regulatory requirements for drug licensing. Pharmaceut Med. 2013; 27 (1): 9-23.

6. Linares Borges A, Milián Vázquez PM, Jiménez Fernández L, Chala Tandrón JM, Alemán Aguilar H, Betancourt Rodríguez BY et al. Interacciones medicamentosas. Acta Farm Bonaerense. 2002; 21 (2): 139-148.

7. Drug Interactions search - MICROMEDEX [Base de datos en Internet]. [consultado 24 de junio de 2020]. Disponible en: https://www.micromedexsolutions.com. [Se requiere suscripción para ver]

8. Masnoon N, Shakib S, Kalisch-Ellett L, Caughey GE. What is polypharmacy? A systematic review of definitions. BMC Geriatr. 2017; 17 (1): 230.
9. Shah BM, Hajjar ER. Polypharmacy, adverse drug reactions, and geriatric syndromes. Clin Geriatr Med. 2012;28 (2):173-186.

10. Ahmed B, Nanji K, Mujeeb R, Patel MJ. Effects of polypharmacy on adverse drug reactions among geriatric outpatients at a tertiary care hospital in Karachi: a prospective cohort study. PLoS One. 2014; 9 (11): e112133.

11. Arce Tintaya A, Castro Luna RC. Caracterización de interacciones medicamentosas potenciales en las prescripciones médicas atendidas a pacientes ambulatorios [Tesis de Licenciatura]. Lima, Perú: Universidad WIENER;2015.

12. Oliván LMG, Hernández CC. Identificación e impacto clínico de las interacciones farmacológicas potenciales en prescripciones médicas del Hospital ISSSTE Pachuca, México. Rev Mex Ciencias Farm. 2006; 37 (4): 30-37.

13. Chahin Ramez C. Interacciones medicamentosas en pacientes hospitalizados en el Servicio de Medicina Interna del Hospital Universitario "Dr. Ángel Larralde": Junio 2014 Diciembre 2015. Arch Venez Farmacol y Ter. 2016; 35 (1): 1-6.

14. Carrasco ZD, Pérez CJ, López SAS, Meljem MJ. Modelo para el análisis de la farmacoterapia en expedientes clínico. Rev CONAMED. 2016; 21 (1): 4-15.

15. Guía de Práctica Clínica de "Interacciones farmacológicas potenciales en la atención del adulto mayor". México: Secretaría de Salud; 2010.

16. Moya CR. Interacciones entre fármacos en una oficina de farmacia comunitaria. Ars Pharm. $2011 ; 52$ (1): 16-22.

17. Bellosta S, Corsini A. Statin drug interactions and related adverse reactions: an update. Expert Opin Drug Saf. 2018; 17 (1): 25-37.

18. Hundal RS, Petersen KF, Mayerson AB, Randhawa PS, Inzucchi S, Shoelson SE et al. Mechanism by which highdose aspirin improves glucose metabolism in type 2 diabetes. J Clin Invest. 2002; 109 (10): 1321-1326.

19. Coe LM, Denison JD, McCabe LR. Low dose aspirin therapy decreases blood glucose levels but does not prevent type I diabetes-induced bone loss. Cell Physiol Biochem. 2011; 28 (5): 923-932.

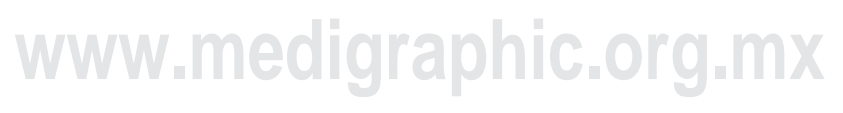

